\title{
Beamline setup \& calibration quality control for synchrotron MX beamlines
}

\author{
D. Aragao, N. Cowieson \\ Diamond Light Source, Harwell Science and Innovation Campus, Chilton, Didcot, OX11 ODE, UK \\ David.Aragao@diamond.ac.uk
}

Macromolecular crystallography instruments around the world are mostly set on a single or handful of configurations. These makes them more predictable and more reliable. At the same time, current throughput demand on MX beamlines squeezes more and more the time for a careful regular maintenance and calibration of the instrument. The latter is extremely important to maximize data quality, protect equipment from failure and detect degradation that can lead to both degradation of performance and unexpected component breakdown down the track with consequence loss of beam hours. Across the world instrument scientists and software engineers have, with success, automated the daily setup \& calibration but often neglected the need for quality control (QC) database recording. Proper QC systems allow a maintenance record of checks with numerous advantages namely: optimizing time by not doing all tests everyday but also guaranteeing that certain tests are done in regular intervals; plot beamline degradation or improvements particularly when new software or hardware is implemented; guarantee that beamline performance is not dependent of synchrotron staff doing the checks because they are all done the same way and recorded the same way; help train new staff into instrument scientist positions and many others. Here we present the next generation of a software tool initially developed at the Australian Synchrotron [1] in Python 2 and using QT 4 but recently re-written with more modern software with Python 3 and QT 5 (DLS internal Gitlab). It is currently in beta tests at Diamond Light Source i04 [2] beamline. The tool attempts to represent the checks currently done (Figure 1a) using visual cues pointing to when the check was last performed as well as provide some guidance on how to do the step-by-step checks. It will then database and file record the result of that check for future reference, tracking and baseline QC (Figure $1 \mathrm{~b}-\mathrm{c}$ ).

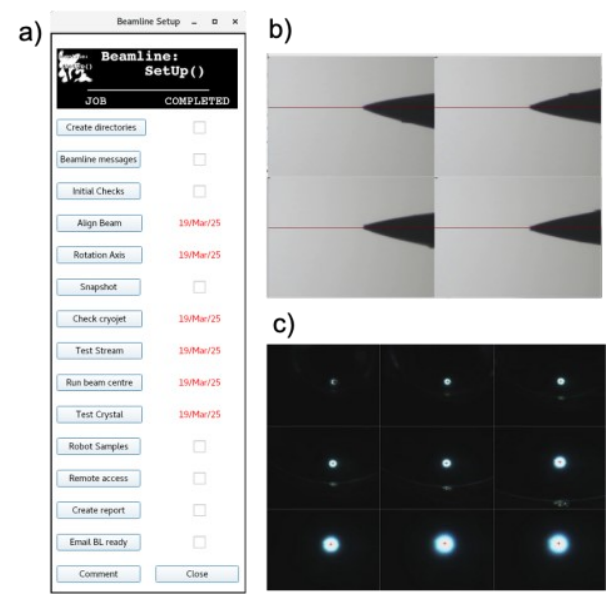

Figure 1. a) Main window showing the different steps as configured for DLS i04 beamline. b) Stored image for Rotation axis QC step showing a needle aligned at orthogonal angles 0 degrees (top left) and 180 degrees (top right); 90 degrees (bottom left) and 270 degrees (bottom right). c) Stored image for Align Beam QC step showing crosshair and beam position for 9 different zoom levels.

\section{[1] https://github.com/AustralianSynchrotron/BeamlineSetup}

[2] R. Flaig et all, Acta Cryst. (2017). A73, a71

\section{Keywords: MX, beamline, calibration, setup, quality control, QC, daily, degradation, beamline, maintenance, instrument science, automation}

We thank many people at both Australian Synchrotron and Diamond Light Source MX beamlines but in particular to Tom CaradocDavies, Daniel Ericsson, Jun Aishima, Stephen Harrop, Kate Smith, Jason Price for suggestions, ideas and code contributions particularly on the initial 2013 version using Python 2 / QT 4 version. 